\title{
CONTRASTE FÔNICO ENCOBERTO ENTRE /T/ E /K/: \\ UM ESTUDO DE CASO DE NORMALIDADE E DE TRANSTORNO FONOLÓGICO
}

\author{
Covert phonic contrast between /t/ and / $/$ : a case study \\ of normality and phonological disorder
}

Larissa Cristina Berti (1), Viviane Cristina de Castro Marino ${ }^{(2)}$

\begin{abstract}
RESUMO
Objetivo: investigar, com auxílio de análise acústica, o estabelecimento do contraste fonológico de produções de um sujeito com transtorno fonológico que apresenta neutralização (pela análise de oitiva) do contraste entre as oclusivas alveolar e velar. Método: foram analisadas as produções das oclusivas /t/ e / $/ \mathrm{k}$ combinadas com as vogais /a/ e /u/ na posição acentuada em dois sujeitos - com e sem transtorno fonológico - ambos do sexo masculino, com faixa etária entre 5 e 6 anos. Os parâmetros fonético-acústicos analisados incluíram a inspeção acústica da forma de onda, os parâmetros relativos às características espectrais do burst; os parâmetros acústicos relativos às características acústicas das vogais adjacentes às oclusivas e os parâmetros acústicos relativos ao padrão temporal. Resultados: nas produções da criança com transtorno fonológico observou-se a presença de contrastes encobertos entre as oclusivas investigadas, marcadas tanto pelo uso inadequado quanto pelo correto das pistas fonéticas, mas com valores insuficientes. Nas produções da criança sem transtorno fonológico observou-se o uso de pelo menos uma pista fonética relativa às principais características de um segmento oclusivo que, com magnitude suficiente, propicia o resgate do contraste entre /t/ e /k/ pelos ouvintes. Nas produções de ambos os sujeitos as pistas fonéticas foram dependentes do contexto vocálico. Conclusão: um determinado contraste fônico pode ser entendido como uma constelação de pistas fonéticas que variam em sua interdependência e significância perceptual.
\end{abstract}

DESCRITORES: Acústica da Fala; Espectrografia; Distúrbios da Fala; Linguagem Infantil; Desenvolvimento Infantil

\section{INTRODUÇÃO}

O transtorno fonológico refere-se a uma desordem lingüística na qual se observa uma

(1) Fonoaudióloga; Docente e Pesquisadora vinculada ao Departamento de Fonoaudiologia da Universidade Estadual Paulista - Unesp, Campus de Marília, no âmbito do Programa de Jovens Pesquisadores em Centros Emergentes de Pesquisa da Fundação de Amparo à Pesquisa do Estado de São Paulo - FAPESP; Doutora em Linguística pelo Instituto de Estudos da Linguagem - IEL da Universidade Estadual de Campinas - UNICAMP.

(2) Fonoaudióloga; Docente vinculada ao Departamento de Fonoaudiologia da Universidade Estadual Paulista UNESP, Campus de Marília; Doutora em Communication Sciences and Disorders pela Universidade da Florida, EUA.

Conflito de interesses: inexistente dificuldade - por parte das crianças - quanto ao conhecimento dos segmentos fonéticos, das regras fonológicas, ou na maneira em que utilizam esse conhecimento. Essa dificuldade pode prejudicar de maneira marcante a inteligibilidade da fala dessas crianças, dependendo do seu grau de gravidade ${ }^{1-6}$. Apesar de diversas pesquisas ter se ocupado em investigar e caracterizar as alterações de produção de fala em crianças com transtornos fonológicos em seus diferentes aspectos ${ }^{7-11}$, o estatuto das pistas fonéticas, ou seja, como essas pistas são manipuladas pelas crianças até atingir o estabelecimento de contrastes fônicos não tem sido objeto de investigação nesses estudos.

Considerando que um determinado contraste fônico pode ser entendido como um conjunto de pistas fonéticas de variada interdependência e 
significância perceptual que se combinam, seu estabelecimento - por parte de uma criança - implica necessariamente que ela não somente utilize as pistas fonéticas mais relevantes (robustas) para diferenciar os segmentos fônicos envolvidos no contraste, como também utilize essas pistas numa magnitudesuficienteque possibiliteseu resgatepelos ouvintes ${ }^{12,13}$.

No tocante à fala de crianças com transtorno fonológico alguns estudos ${ }^{14-20}$ têm mostrado, com o uso de uma metodologia instrumental (análise acústica e/ou articulatória), que muitas das produções julgadas como idênticas em uma análise de outiva (neutralização fônica), tratam-se, na verdade, do chamado "contraste fônico encoberto". A expressão "contraste fônico encoberto" (covert contrast) é utilizada para descrever o que é categorizado como contrastes fônicos imperceptíveis auditivamente, mas detectáveis acústica e/ou articulatoriamente ${ }^{21}$.

Nos estudos citados acima os contrastes fônicos encobertos identificados nas produções das crianças com transtornos fonológicos são explicados, fundamentalmente, devido aos seguintes fatores: a) uso de pistas fonéticas não robustas para a língua em questão (ou seja, uso inapropriado de pistas fonéticas) e/ou; b) uso de pistas fonéticas robustas (apropriadas) para a língua em questão, mas em magnitudes não previstas: com valores insuficientes ou exacerbados ${ }^{22}$.

Frente ao exposto, a identificação de contrastes fônicos encobertos somente é possível com a utilização de uma metodologia instrumental (acústica e/ou articulatória), na qual possibilita apreender quais e, além disso, em que magnitude os parâmetros fonético-acústicos são utilizados pelas crianças no estabelecimento de um determinado contraste fônico.

A hipótese que se pretende perseguir neste estudo é a de que muitas das "trocas" na fala de crianças com transtorno fonológico não se trata de uma substituição categórica entre os dois segmentos, mas de um contraste fônico encoberto.

Com efeito, a proposta do presente estudo é investigar, com auxílio de análise acústica, o estabelecimento do contraste fonológico de produções de um sujeito com o diagnóstico de transtorno fonológico que apresenta neutralização (a partir da análise de oitiva) do contraste entre as oclusivas surdas, alveolar e velar. Ou seja, apresenta posteriorização da oclusiva alveolar [t], realizando-a de forma semelhante, auditivamente, à oclusiva velar [k]. Especificamente, o presente estudo pretende responder quais, e em que magnitude, os parâmetros fonético-acústicos são utilizados no estabelecimento do contraste entre $/ \mathrm{t} / \mathrm{e} / \mathrm{k} /$.

\section{MÉTODO}

Participaram desse estudo duas crianças, do sexo masculino, entre 5 e 6 anos de idade, falantes do Português Brasileiro. Uma das crianças apresentava o diagnóstico de transtorno fonológico - apresentando, a partir da análise de oitiva, uma neutralização fônica entre /t/ e /k/; e a outra criança apresentava desenvolvimento típico de linguagem. No presente estudo, a criança com desenvolvimento típico de linguagem foi designada como S1, enquanto a criança com o diagnóstico de transtorno fonológico foi designada como S2.

O corpus foi constituído por palavras dissílabas paroxítonas que combinavam as oclusivas /t/ e /k/ com as vogais /a/ e /u/ na posição acentuada, a saber: taco, tuba, capa e cuca. O contexto da vogal /i/ foi excluído pelo fato da oclusiva /t/ diante dessa vogal ser produzido como africada (/t $\int /$ ) na região onde foi desenvolvida a pesquisa.

O procedimento experimental consistiu em solicitar das crianças cinco repetições de cada uma das palavras no interior da seguinte frase veículo: "Fale palavra alvo bem bonito", por meio de uma atividade lúdica, computando um total de 40 ocorrências, favorecendo, desse modo, a realização da análise acústica e posterior tratamento estatístico dos dados obtidos: 05 (repetições) $\times 2$ (vogais) $X 2$ (oclusivas) $X 2$ (sujeitos) $=40$ ocorrências.

Adicionalmente, o uso da frase veículo teve como objetivo propiciar um maior controle da curva entonacional da produção de cada palavra do corpus, tentando assim, evitar a curva ascendente característica da produção obtida por meio de repetição isolada em forma de lista de palavras.

Cada criança foi gravada numa sala tratada acusticamente com equipamento de alta fidelidade. Utilizou-se para análise dos dados o software PRAAT ${ }^{23}$ e para análise estatística o software STATISTICA 24.

Os parâmetros fonético-acústicos adotados na análise foram: (1) inspeção acústica da forma de onda; (2) parâmetros relativos às características espectrais do burst: pico espectral e momentos espectrais (centróide, variância, assimetria e curtose); (3) parâmetros relativos às características acústicas das vogais adjacentes às oclusivas: medidas das freqüências da transição formântica oclusivas/vogais (F1, F2 e F3) e trajetórias formânticas das vogais (F1, F2 e F3) e; (4) parâmetros relativos ao padrão temporal: medidas de duração (absoluta e relativa) da closura, burst e transição formântica.

Cabe ressaltar que do ponto de vista acústico as oclusivas caracterizam-se a partir de três parâmetros principais: closura, burst e transição formântica. 
A closura equivale ao tempo em que os articuladores permanecem bloqueados. O burst ou soltura refere-se a um transiente acústico de duração reduzida e, por fim, a transição formântica diz respeito à transição articulatória da oclusiva para vogal adjacente, marcada por uma transição acústica na forma dos formantes ${ }^{25,26}$.

Uma descrição mais detalhada dos parâmetros acústicos utilizados na análise encontra-se a seguir:

1) inspeção acústica da forma de onda, diz respeito à apreensão visual das três partes que caracterizam as oclusivas: closura, burst e transição formântica;

2) parâmetros relativos às características espectrais do burst: (a) pico espectral ou maior pico de energia do espectro: refere-se à posição do pico de maior energia do espectro das freqüências dadas pela FFT (gráfico resultante da decomposição de sons complexos em um conjunto de senóides de diferentes amplitudes e freqüências) e b) momentos espectrais: referem-se a uma métrica quantitativa baseada na análise estatística do espectro ${ }^{27}$. Isto é, são calculadas a média (centróide), a variância, a assimetria e a curtose do espectro. Tais medidas tentam incorporar tanto informações locais do espectro (como, por exemplo, o pico espectral) quanto informações mais globais do espectro (como a forma espectral).

Particularmente, o centróide corresponde ao primeiro momento da distribuição espectral, obtido por meio do cálculo da média ponderada da intensidade dos componentes de freqüência de um espectro FFT que apontam para um centro de gravidade. Já a variância (segundo momento espectral) corresponde à variância das freqüências dadas pelo espectro e descreve a variabilidade da distribuição em relação à média. A assimetria (terceiro momento espectral) corresponde a uma medida que indica como é a distribuição das freqüências em torno da média. Uma assimetria igual a zero indica uma distribuição simétrica em torno da média. Já uma assimetria positiva sugere uma inclinação negativa com concentração de energia em freqüências mais baixas; enquanto uma assimetria negativa está associada com inclinação positiva e predominância de energia em freqüências mais altas ${ }^{28}$. Quanto a curtose (quarto momento espectral), este é um indicador do pico da distribuição das freqüências no espectro. Uma curtose positiva indica picos relativamente altos no espectro, ou ainda, quanto maior o valor da curtose mais picos estão presentes na distribuição do espectro. Já uma curtose negativa, indica que a distribuição apresenta picos mais achatados, ou então um espectro sem picos bem definidos ${ }^{28}$;

3) parâmetros relativos às características acústicas das vogais adjacentes às oclusivas - transição formântica de F1, F2 e F3: refere-se à tendência de inclinação da trajetória formântica na transição do som investigado com a vogal seguinte. Essa tendência pode ser de elevação ou declínio e;

4) parâmetros relativos ao padrão temporal: (a) duração absoluta: refere-se à duração de um segmento, ou de partes dele, em milisegundos e (b) duração relativa: refere-se à duração de um segmento, ou de partes dele, em relação à duração total da palavra na qual está inserido, ou do próprio segmento, quando nos referirmos às partes específicas do mesmo (como closura, burst e/ou transição formântica).

Este estudo foi aprovado pelo Comitê de Ética em Pesquisa da Faculdade de Filosofia em Ciência - FFC/Unesp - Marília - São Paulo, sob o protocolo de número 3499/2006. Os pais ou responsáveis pelos sujeitos analisados assinaram o Termo de Consentimento Pós-Informação.

No presente estudo, para os dados de duração relativa foi utilizada uma análise estatística não paramétrica com o uso dos testes Mann-Whitney, para amostras independentes e; Wilcoxon, para amostras dependentes. Já para os demais parâmetros acústicos foi utilizada uma análise estatística paramétrica com o uso do Teste T pareado para amostras dependentes. Os testes estatísticos utilizados foram aplicados para as produções seguidas de /a/ e /u/ separadamente. Foram considerados estatisticamente significantes os resultados com valores de $p$ inferiores a $0,05(p<0,05)$ e marginais os resultados com valores de $p$ entre $0,05 \mathrm{e}$ $0,1(0,05<p<0,1)$. Nas tabelas, os valores estatisticamente significantes foram colocados em negrito.

\section{RESULTADOS}

Para responder os objetivos propostos nesse estudo, ou seja, quais e, em que magnitude, os parâmetros fonético-acústicos são utilizados para o estabelecimento do contraste entre /t/ e / $/ \mathrm{k} /$ num sujeito com diagnóstico de transtorno fonológico, os resultados serão descritos em função de cada parâmetro acústico adotado em nossa análise.

Iniciou-se a análise acústica dos dados a partir da inspeção visual da forma de onda, especificamente, a partir da inspeção visual das três porções que caracterizam as oclusivas - closura, burst e 
transição formântica - de todas as produções de /t/ e / $/$ / seguidos das vogais /a/ e /u/ dos dois sujeitos (S1 e S2).

Dentre as 10 ocorrências de /t/ nas produções de S2 (sujeito com diagnóstico de transtorno fonológico), apreendidas auditivamente como / $/ \mathrm{em}$ ambos os contextos vocálicos, constatou-se que em

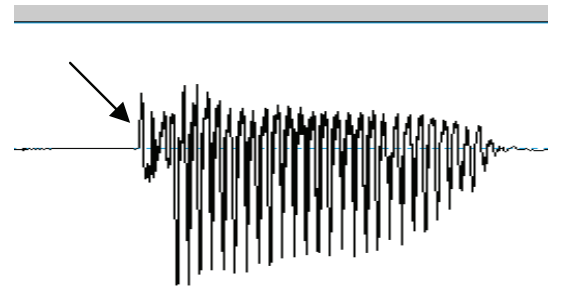

(a)

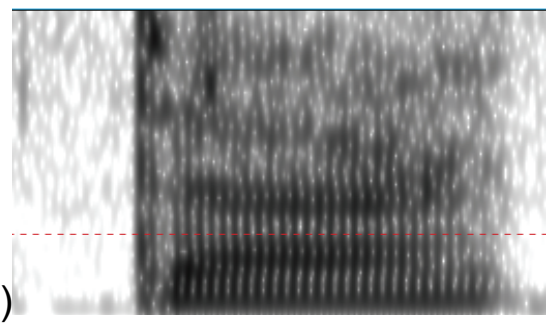

$40 \%$ dessas produções a ocorrência de um fenômeno extremamente interessante, a saber: duplos burts, ou seja, a presença de dois transientes acústicos, de amplitudes distintas, com durações extremamente reduzidas. A Figura 1 ilustra a inspeção acústica de um /t/ típico e um /t/ com a presença de duplos bursts.



(b)

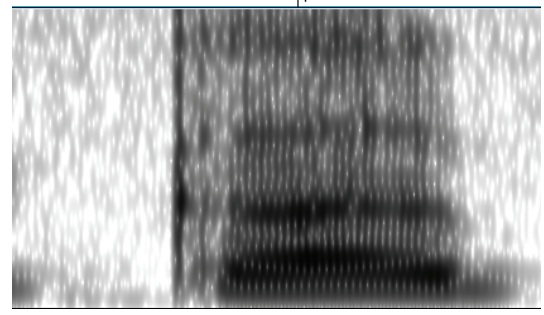

Figura 1 - (a) inspeção acústica de um único burst na produção de um /t/ típico pelo sujeito com produções típicas (S1) e (b) presença de duplos bursts na produção de um /t/ pelo sujeito com produções desviantes (S2)

Detectada a presença dos duplos bursts, realizou-se uma análise espectral de cada um dos estouros a fim de identificar o maior pico de energia dado pelo espectro. Surpreendentemente, nas produções de S2 que foram detectadas ocorrências de duplos bursts, o valor do pico do primeiro estouro apontou para características acústicas sugestivas de $\mathrm{um} / \mathrm{k} /$ (valor médio de $1540 \mathrm{~Hz}$ ), enquanto o valor do pico do segundo estouro apontou para características acústicas sugestivas de um /t/ (valor médio de $2847,31 \mathrm{~Hz}$ ). A Figura 2 ilustra a análise espectral de cada explosão separadamente. Já nas demais produções de S2 (no contexto da vogal /u/) e, ainda, em todas as produções de S1 não foi detectada a presença de duplos bursts, a partir da inspeção da forma de onda. (a)

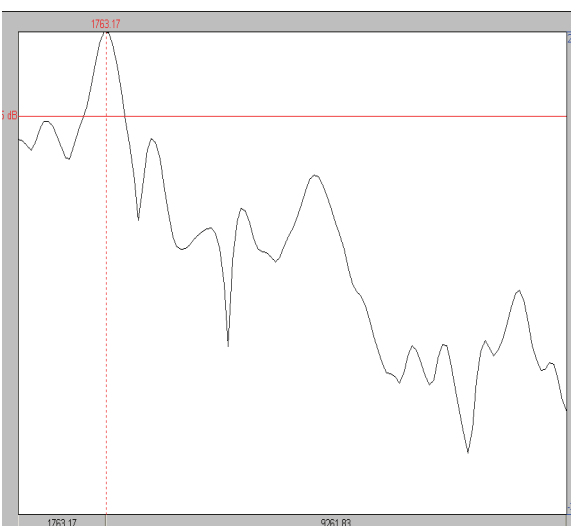

(b)

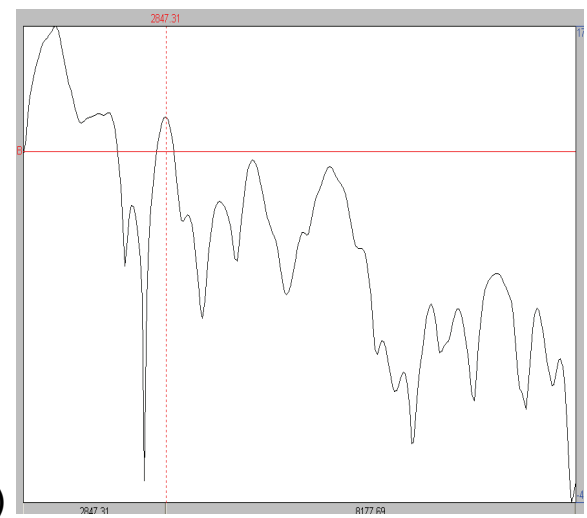

Figura 2 - Análise espectral de cada um dos estouros, separadamente. A flecha disposta no gráfico (a) sugere característica espectral de $/ \mathrm{k} /(1763,17 \mathrm{~Hz})$ enquanto a flecha disposta no gráfico (b) indica característica espectral de /t/ $(1640 \mathrm{~Hz})$ 
Em relação aos parâmetros acústicos relativos às características espectrais do burst, os resultados encontrados estão dispostos nas Tabelas 1 e 2. Destaca-se, dentre os dados dispostos nestas tabelas, três aspectos: (i) nem todos os parâmetros foram sensíveis para distinguir / $/ \mathrm{t} / \mathrm{e} / \mathrm{k} /$, mesmo nas produções típicas de S1; (ii) os parâmetros acústicos são dependentes do contexto vocálico em ambos os sujeitos (S1 e S2); e (iii) o pico espectral foi sensível para resgatar o contraste entre /t/ e /k/ na produção de S2, julgada auditivamente como idêntica.

Tabela 1 - Valores médios das características espectrais do burst no contexto da vogal /a/

\begin{tabular}{|c|c|c|c|c|c|c|}
\hline \multirow{3}{*}{$\begin{array}{c}\text { Características espectrais } \\
\text { burst }\end{array}$} & \multicolumn{6}{|c|}{ Contexto da vogal /a/ } \\
\hline & \multicolumn{3}{|c|}{ S1 (produções típicas) } & \multicolumn{3}{|c|}{ S2 (produções desviantes) } \\
\hline & $\begin{array}{c}/ \mathrm{t} / \\
\text { (média) }\end{array}$ & $\begin{array}{c}\text { /k/ } \\
\text { (média) }\end{array}$ & p-value & $\begin{array}{c}/ \mathrm{t} / \\
\text { (média) }\end{array}$ & $\begin{array}{c}/ \mathbf{k} / \\
\text { (média) }\end{array}$ & p-value \\
\hline Pico espectral (Hz) & 4529,74 & 2124,71 & $0,03^{*}$ & 2506,85 & 1696,62 & $0,02^{*}$ \\
\hline Centróide $(\mathrm{Hz})$ & 1888,09 & 1704,15 & 0,73 & 1098,79 & 1303,81 & 0,25 \\
\hline Variância (MHz) & 3394,96 & 2727,7 & 0,46 & 888,2 & 2029,4 & 0,10 \\
\hline Assimetria & 2,23 & 2,09 & 0,82 & 2,93 & 2,59 & 0,61 \\
\hline Curtose & 5,34 & 7,44 & 0,40 & 17,77 & 10,49 & 0,23 \\
\hline
\end{tabular}

${ }^{*}$ Valores estatisticamente significantes $(p<0,05)$

Tabela 2 - Valores médios das características espectrais do burst no contexto da vogal /u/

\begin{tabular}{|c|c|c|c|c|c|c|}
\hline \multirow{3}{*}{$\begin{array}{c}\text { Características espectrais } \\
\text { burst }\end{array}$} & \multicolumn{6}{|c|}{ Contexto da vogal /u/ } \\
\hline & \multicolumn{3}{|c|}{ S1 (produções típicas) } & \multicolumn{3}{|c|}{ S2 (produções desviantes) } \\
\hline & $\begin{array}{c}\text { /t/ } \\
\text { (média) }\end{array}$ & $\begin{array}{c}/ \mathbf{k} / \\
\text { (média) }\end{array}$ & p-value & $\begin{array}{c}\text { /t/ } \\
\text { (média) }\end{array}$ & $\begin{array}{c}\text { /kJ } \\
\text { (média) }\end{array}$ & $p$-value \\
\hline Pico espectral $(\mathrm{Hz})$ & 5492,49 & 5444,8 & 0,92 & 5341,86 & 5454,35 & 0,58 \\
\hline Centróide $(\mathrm{Hz})$ & 342,73 & 539,5 & $0,04^{*}$ & 554,066 & 721,08 & 0,18 \\
\hline Variância (MHz) & 1036,61 & 1208,82 & 0,77 & 379,53 & 841,21 & 0,49 \\
\hline Assimetria & 5,838 & 10,132 & 0,14 & 7,016 & 7,804 & 0,78 \\
\hline Curtose & 41,472 & 152,274 & 0,13 & 121,68 & 136,982 & 0,88 \\
\hline
\end{tabular}

*Valores estatisticamente significantes $(p<0,05)$

No tocante aos parâmetros relativos às características acústicas das vogais adjacentes - transição formântica de F1, F2 e F3 - os resultados obtidos na análise acústica estão sintetizados nas Tabelas 3 e 4.

Com base nos resultados dispostos nas Tabelas 3 e 4, observa-se, mais uma vez, que os parâmetros relativos às características das vogais adjacentes também são dependentes do contexto vocálico, ao diferenciar as duas oclusivas nas produções dos dois sujeitos.

Finalmente, no que se refere aos parâmetros referentes ao padrão temporal das duas principais partes dos segmentos oclusivos (closura e burst), verifica-se que - em termos absolutos - somente $\mathrm{S} 1$ diferenciou /t/ e /k/ no contexto da vogal /u/, tanto pela duração absoluta da closura quanto pela duração absoluta do burst, com valores de $p=0,04 \mathrm{e}$ $p=0,05$, respectivamente.

No entanto, quando foram considerados os valores de duração relativos, constata-se que no contexto da vogal /a/, tanto S1 quanto S2 diferenciam /t/ e / $/ \mathrm{k}$ pela medida relativa da closura, com valores de $p=0,04$ e $p=0,05$, respectivamente. Somente $S 1$ distinguiu /t/ e / $/$ pelo valor relativo do burst $(p=0,04)$. 
Tabela 3 - Valores médios das características acústicas da vogal /a/ adjacente

\begin{tabular}{|c|c|c|c|c|c|c|}
\hline \multirow{3}{*}{$\begin{array}{l}\text { Características acústicas } \\
\text { das vogais adjacentes }\end{array}$} & \multicolumn{6}{|c|}{ Contexto da vogal /a/ } \\
\hline & \multicolumn{3}{|c|}{ S1 (produções típicas) } & \multicolumn{3}{|c|}{ S2 (produções desviantes) } \\
\hline & $\begin{array}{c}/ \mathrm{t} / \\
\text { Média }\end{array}$ & $\begin{array}{c}/ \mathbf{k} / \\
\text { Média }\end{array}$ & p-value & $\begin{array}{c}\text { /t/ } \\
\text { Média }\end{array}$ & $\begin{array}{c}/ \mathbf{k} / \\
\text { Média }\end{array}$ & $p$-value \\
\hline Transição Formântica F1(Hz) & 716,8 & 707,05 & 0,90 & 599,31 & 576,22 & 0,55 \\
\hline Transição Formântica F2(Hz) & 1985,42 & 1768,96 & 0,11 & 1630,52 & 1707,5 & 0,08 \\
\hline Transição Formântica F3(Hz) & 3254,38 & 2623,32 & $0,00^{*}$ & 2938,84 & 2838,8 & 0,13 \\
\hline
\end{tabular}

*Valores estatisticamente significantes $(p<0,05)$

Tabela 4 - Valores médios das características acústicas da vogal /u/ adjacente

\begin{tabular}{|c|c|c|c|c|c|c|}
\hline \multirow{3}{*}{$\begin{array}{l}\text { Características acústicas } \\
\text { das vogais adjacentes }\end{array}$} & \multicolumn{6}{|c|}{ Contexto da vogal /u/ } \\
\hline & \multicolumn{3}{|c|}{ S1 (produções típicas) } & \multicolumn{3}{|c|}{ S2 (produções desviantes) } \\
\hline & $\begin{array}{c}1 / \mathrm{t} / \\
\text { (média) }\end{array}$ & $\begin{array}{c}/ \mathbf{k} / \\
\text { (média) }\end{array}$ & p-value & $\begin{array}{c}/ \mathrm{t} / \\
\text { (média) }\end{array}$ & $\begin{array}{c}/ \mathbf{k} / \\
\text { (média) }\end{array}$ & p-value \\
\hline Transição Formântica F1(Hz) & 445,40 & 532,38 & 0,17 & 437,6 & 414,92 & 0,24 \\
\hline Transição Formântica F2(Hz) & 1946,08 & 1652,52 & $0,05^{\star}$ & 1268,3 & 1442,87 & 0,23 \\
\hline Transição Formântica F3(Hz) & 3235,2 & 2808,30 & $0,02^{*}$ & 3257,2 & 2633,52 & $0,05^{\star}$ \\
\hline
\end{tabular}

*Valores estatisticamente significantes $(p<0,05)$

Já no contexto da vogal / $\mathrm{u} /$, somente $\mathrm{S} 1$ diferenciou os dois segmentos fônicos analisados tanto pela medida relativa do burst quanto pela medida relativa da closura - com valores de $p=0,04$ nos dois casos. As Figuras 3 e 4 ilustram os resultados descritos acima para o contexto da vogal /a/.

Na próxima seção são apresentadas as hipóteses explicativas dos resultados observados.

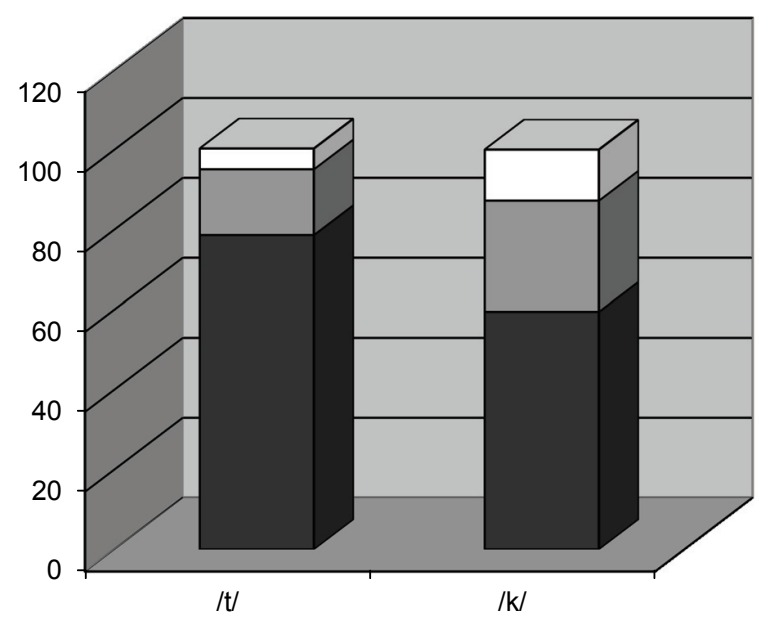

๑\% da transição formântica

$\square \%$ do burst

$\square \%$ da closura

Figura 3 - Comparação entre as durações relativas de /t/ e /k/ no contexto da vogal /a/ nas produções do sujeito com produções típicas (S1) 


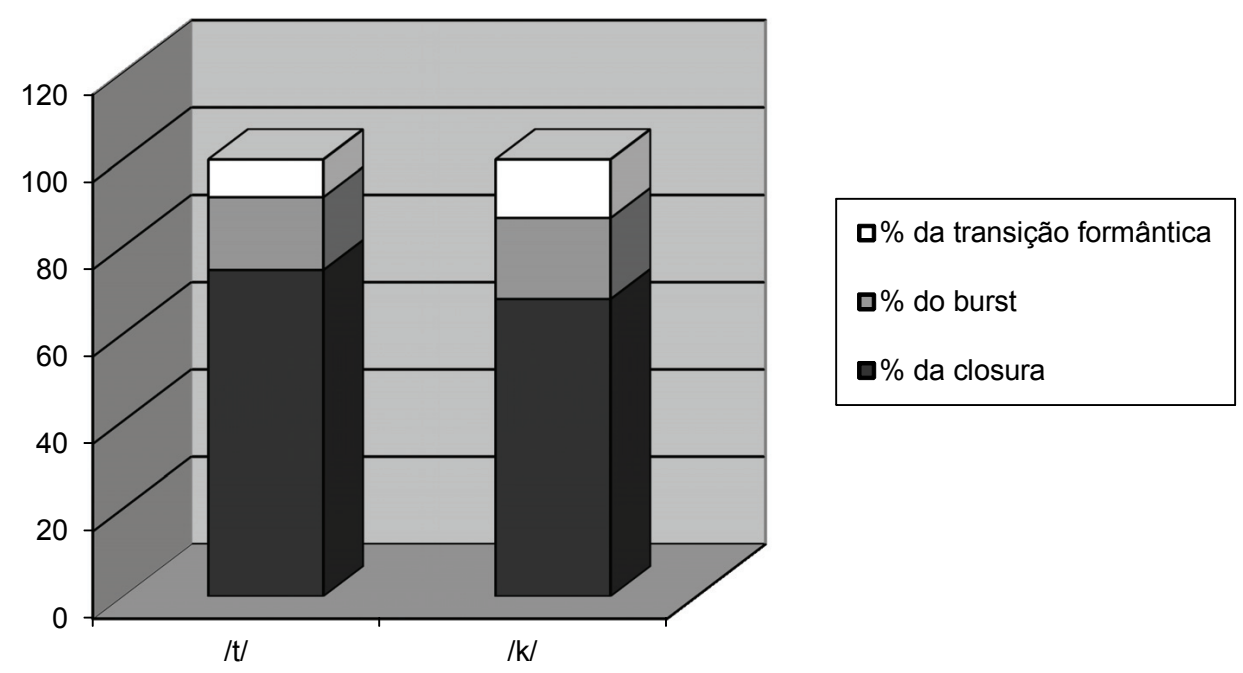

Figura 4 - Comparação entre as durações relativas de /t/ e /k/ no contexto da vogal /a/ nas produções do sujeito com produções desviantes (S2)

\section{DISCUSSÃO}

Um primeiro destaque a ser feito em relação aos resultados refere-se ao uso dos parâmetros fonético-acústicos por S1 e S2 no estabelecimento do contraste entre $/ \mathrm{t} / \mathrm{e} / \mathrm{k} /$. Verificou-se que nem todos os parâmetros adotados na análise acústica foram sensíveis para resgatar a diferença entre /t/ e /k/, mesmo nas produções de S1 (julgadas auditivamente como típicas) e, além disso, esses parâmetros foram dependentes do contexto vocálico.

Especificamente, observou-se que S1 (sujeito com produções típicas) diferenciou /t/ e / $/ \mathrm{k} /$ no contexto da vogal /a/ pelos seguintes parâmetros: pico espectral; transição formântica de F3 e durações relativas da closura e burst. Já no contexto da vogal /u/, S1 diferenciou o contraste investigado pelos parâmetros acústicos centróide, transições formânticas de F2 e F3, além das medidas de duração absoluta e relativa da closura e do burst.

Destaca-se que, embora S1 não tenha utilizado todos os parâmetros acústicos adotados na análise acústica, tanto no contexto da vogal /a/ quanto no contexto da vogal /u/, este sujeito utilizou pelo menos um dos parâmetros acústicos relacionados às principais características das oclusivas descritas na literatura ${ }^{25,26,28-30}$, ou seja, características espectrais do burst (pico espectral e centróide); características acústicas das vogais adjacentes (transição formântica de F2 e F3) e características temporais (duração relativa e absoluta da closura e burst).

Desse modo, hipotetiza-se que embora S1 não utilize todos os parâmetros acústicos adotados para diferenciar /t/ e / $/ \mathrm{k}$, o uso de pelo menos um parâmetro acústico relacionado às três principais partes de um segmento oclusivo (característica espectral do burst, transição formântica e padrão temporal da oclusiva), em uma magnitude suficiente, é o que possibilita o resgate de suas produções como sendo típicas por parte dos ouvintes.

Esse achado corrobora com os encontrados em estudos anteriores ${ }^{12,13,22}$ que consideram um determinado contraste fônico como um conjunto de pistas fonéticas, de variada interdependência e significância perceptual, que se combinam de forma a resultar em um determinado contraste fônico. Desse modo, o estabelecimento de um determinado contraste por parte de uma criança - implica necessariamente - que ela não somente utilize as pistas fonéticas mais relevantes (robustas) para diferenciar os segmentos fônicos envolvidos no contraste, como também utilize essas pistas numa magnitude suficiente que possibilite seu resgate pelos ouvintes.

Um segundo destaque a ser feito dos resultados obtidos refere-se às produções de S2 (criança com diagnóstico de transtorno fonológico) que, do ponto de vista perceptivo-auditivo, apresentava uma neutralização fônica entre $/ \mathrm{t} / \mathrm{e} / \mathrm{k} /$. Contrariamente, a partir da análise acústica constatou-se que S2 não apresenta uma neutralização fônica propriamente dita, uma vez que essa criança já utiliza alguns parâmetros acústicos para distinguir /t/ e /k/ . Particularmente, S2 distinguiu /t/ e / k/ no contexto da vogal /a/ pelo pico espectral e duração relativa da closura; e no contexto da vogal/u/, apenas pela transição formântica de F3. A partir desses achados pode-se afirmar que S2 já iniciou uma distinção entre $/ \mathrm{t} / \mathrm{e} / \mathrm{k} /$, em termos de produção, caracterizando o chamado contraste fônico encoberto ${ }^{21}$. 
Destaca-se, ainda, o uso insuficiente de parâmetros acústicos nas produções de S2 para distinguir /t/ e /k/ em ambos os contextos vocálicos, corroborando com diversos estudos que descreveram a presença de contraste fônico encoberto nas produções de crianças com diagnóstico de transtorno fonológico ${ }^{17,18}$.

Finalmente, um terceiro aspecto a ser ressaltado na análise das produções de S2 diz respeito à identificação de duplos bursts. Observou-se que em $40 \%$ das produções de /t/ apreendida auditivamente como $/ \mathrm{k} /$ ocorreram duplos bursts, onde cada explosão apresentou característica espectral distinta. Ou seja, a primeira explosão - de maior amplitude - apresentou característica espectral indicativa de um $/ \mathrm{k} /$; e a segunda explosão - de menor amplitude - apresentou característica espectral sugestiva de um /t/ (conforme ilustrado nos Gráficos 1 e 2). Esses resultados indiciam que S2 está realizando, em algumas tentativas de produção de /t/ (40\%) no contexto da vogal /a/, uma dupla articulação quase simultaneamente, isto é, uma produção com o uso de dois gestos articulatórios: um gesto de corpo de língua e outro de ponta de língua, com magnitudes diferentes.

Embora não tenha sido encontrada na literatura nenhuma descrição de duplos bursts na produção de fala de crianças com desenvolvimento típico e/ ou desviante da linguagem, há três estudos ${ }^{30-32}$ que reportam a constatação de duplos bursts em erros de produção de fala induzidos (envolvendo justamente a produção de /t/ e / $/ \mathrm{k}$ ) em adultos.

Nesses estudos, os autores induziram os adultos a cometerem erros de produção de fala envolvendo /t/ e /k/ (como, por exemplo, solicitando a produção repetida e com um aumento gradativo de velocidade da sequência "cop" e "top"). Estes autores constataram, a partir de uma análise instrumental articulatória (articulografia), a presença de dupla articulação - um gesto de corpo de língua e outro de ponta de língua, com magnitudes diferentes - nos erros de produção de fala interpretados como sendo uma neutralização fônica entre esses segmentos, quando analisados somente a partir de uma análise de oitiva. Tais autores descreveram esse fenômeno como sendo erros de produção de fala caracterizados por uma inserção gestual ${ }^{32}$.

De modo semelhante aos adultos investigados pelos autores citados acima, supõe-se que muitas das produções de S2 (especificamente, em 40\% das tentativas de produção de /t/ em contexto da vogal /a/) indiciam, na verdade, produções intermediárias entre as categorias de /t/ e / $/$, caracterizadas por erros de intrusão gestual.

\section{CONCLUSÃO}

A partir dos resultados obtidos no presente estudo, verificou-se que as neutralizações fônicas de S2 tratam-se - na verdade - de contrastes encobertos entre as duas oclusivas investigadas, caracterizados especialmente por um conjunto de pistas fonéticas, tais como: duplos bursts, parâmetros de duração, pico espectral, centróide e transição formântica. Isso significa dizer que S2 parece estar avançando em direção ao domínio gradual dos parâmetros fonético-acústicos faltantes para o estabelecimento do contraste entre as oclusivas investigadas.

No tocante as produções típicas de S1, constatou-se que apesar dessa criança não utilizar todos os parâmetros acústicos para marcar a diferença entre $/ t / \mathrm{e} / \mathrm{k} /$, o uso de pelo menos uma pista fonética relativa às principais características de um segmento oclusivo com uma magnitude suficiente, propicia o resgate de tal contraste por parte dos ouvintes.

Quanto à consideração do contexto vocálico no estabelecimento do contraste investigado, observou-se que tanto nas produções de S1 quanto nas produções de $S 2$, as pistas fonéticas foram dependentes do contexto vocálico. Particularmente, o contexto da vogal /a/ parece favorecer o estabelecimento do contraste investigado por essas crianças.

Portanto, um determinado contraste fônico pode ser visto como uma constelação de pistas fonéticas que variam em sua interdependência e em sua significância perceptual. Conseqüentemente, o momento em que a criança começa produzir um determinado contraste fônico e o momento em que esse contraste passa a ser perceptível ao ouvinte não precisa ser sincrônico. Neste processo de estabelecimento do contraste fônico a diferenciação, o ajuste e a coordenação de gestos articulatórios desempenham um papel fundamental.

\section{AGRADECIMENTOS}

À Fundação de Amparo à Pesquisa do Estado de São Paulo (FAPESP), pelo apoio concedido no processo no 06/61816-4. 


\begin{abstract}
Purpose: to investigate, using an acoustic analysis, the establishment of phonological contrast between alveolar and velar stop sounds in a child with phonological disorders that neutralizes this contrast. Method: productions of stop /t/ and $/ \mathrm{k} /$ sounds combined with the vowels $/ \mathrm{a} / \mathrm{and} / \mathrm{u} / \mathrm{in}$ the stressed position were investigated in two male children between 5 and 6-year old, one with phonological disorders and the other with no disorder. The analyzed acoustic parameters included acoustic waveform inspection, acoustic parameters related to spectral burst characteristics, acoustic parameters related to the characteristics of vowels adjacent to occlusive sounds and acoustic parameters related to temporal patterns of the productions. Results: findings from this study indicated presence of covert contrast in the two stop sounds produced by the child with phonological disorders, marked by both inadequate phonetic cues and adequate phonetic cues, but with insufficient values. Productions of the child without phonological contrast evidenced the use of at least one phonetic cue related to the primary acoustic characteristics of stop segments, which allows for auditory perception of the contrast between $/ \mathrm{t} /$ and $/ \mathrm{k} /$ sounds. The productions of both children - with and without phonological disorders - were vocalic context dependent. Conclusion: a determined phonetic contrast can be understood as a constellation of phonetic cues that vary in terms of their interdependency and/ or perceptual significance.
\end{abstract}

KEYWORDS: Speech Acoustics; Spectrography; Speech Disorders; Child Language; Child Development

\section{REFERÊNCIAS}

1. Grunwell P. Processes of phonological change in developmental speech disorders. Clin Linguist Phon. 1992; 6:101-22.

2. Rvachew S, Grawburg M. Correlates of phonological in preschoolers with speech sound disorders. Journal of Speech, Language and Research. 2006; 49: 74-87.

3. Wertzner HF, Alves RR, Ramos ACO. Análise do desenvolvimento das habilidades diadococinéticas orais em crianças normais e com transtorno fonológico. Rev. Soc. Bras. Fonoaudiol. 2008;13(2):136-42.

4. Wertzner HF. Distúrbio Fonológico. In: Andrade CRF, Marcondes E. (Org.). Fonoaudiologia em Pediatria. 1. ed. São Paulo: Savier, 2003. p.70-8.

5. Preston J, Edwards ML. Phonological Awareness and Types of Sound Errors in Preschoolers With Speech Sound Disorders. J Speech Lang Hear Res. 2010; 53(1): $44-60$.

6. Wertzner HF, Amaro L, Galea DES. Phonological performance measured by speech severity indices compared with correlated factors. SP Med J. 2007; 125(6):309-14.

7. Mezzomo CL, Mota HB, Dias RF, Giacchini V. O uso da estratégia de alongamento compensatório em crianças com desenvolvimento fonológico normal e desviante. Letras Hoje. 2008; 43:35-41.

8. Mota HB, Kaminski TI, Flores M, Athayde ML. Alterações no vocabulário expressivo de crianças com desvios fonológicos. Rev. Soc. Bras. Fonoaudiol. 2009;14:41-7.

9. Wertzner HF, Silva LM. Velocidade de fala em crianças com e sem transtorno fonológico. Pró-Fono (Impresso) 2009; 21:19-24.

10. Sutherland D, Gillon GT. Development of phonological representations and phonological awareness in children with speech impairment. International Journal Lang Commun Disord. 2007; 42(2):229-50.

11. Rvachew S, Chiang PY, Evans N. Characteristics of speech errors produced by children with and without delayed phonological awareness skills. Lang Speech Hear Serv Schools; 2007; 38(1):60-71.

12. Scobbie JM. Interactions between the acquisition of phonetics and phonology. In: Gruber C, Higgins D, Olson K, Wysocki T. (eds.) Papers from the 34th Annual Regional Meeting of the Chicago Linguistic Society, Volume II: The Panels; 1998: 343-58. Chicago: Chicago Linguistics Society.

13. Cristià A, Seidl A. Is Infants' Learning of Sound Patterns Constrained by Phonological Features? Language Learning and Development. 2008: 4(3): $203-27$.

14. Hewlett N, Waters D. Gradient change in the acquisition of phonology. Clin Linguist Phon. 2004;18(6-8):523-33.

15. Langstrof $C$. Acoustic evidence for a pushchain shift in the intermediate period of New Zealand English. Language Variation and Change. 2006;18:141-64. 
16. Lowenstein JH, Nittrouer S. Patterns of acquisition of native voice onset time in English-learning children. J. Acoust. Soc. Am. 2008;124(8):1180-91. 17. Rodrigues LL, Freitas MC, Albano EC, Berti LC. Acertos gradientes nos chamados erros de pronúncia. Revista Letras. 2008; 36:85-112.

18. Berti LC, Albano EC. Revisiting Phonological Disorder: An Analysis of Speech Production and Perception. Studies Languages. 2008; 44:22-32.

19. Li F, Edwards J, Beckman M. Contrast and covert contrast: the phonetic development of voiceless sibilant fricatives in English and Japanese toddlers. Journal of Phon. 2009; 37(2):189-211.

20. Munson B, Edwards J, Schellinger SK, Beckman M, Meyer M. Deconstructing phonetic transcription: covert contrast, perceptual bias, and an extraterrestrial view of Vox Humana. Clin Linguist Phon. 2010;24(4-5):245-60.

21. Hewlett N. Acoustic properties of $/ \mathrm{k} /$ and $/ \mathrm{t} / \mathrm{in}$ normal and phonological disordered speech. Clin Linguist Phon. 1988;2:29-45.

22. Scobbie JM, Gibbon F, Hardcastle WJ, Fletcher $P$. Covert contrast as a stage in the acquisition of phonetics and phonology. In: Broe M, Pierrehumbert $\mathrm{J}$ editors. Papers in Laboratory Phonology V: Language Acquisition and the Lexicon. 2000; 5(1):194-207.
23. Boersman $P$, Weenink D. Praat: doing phonetics by computer (Version 5.0.30) Avaliable from: http:// www.praat.org/ (Nov 2008).

24. StatSoft, Inc. STATISTICA (data analysis software system), version 6.0. (2001).

25. Kent RD, Read C. (1992). The acoustic analysis of speech. San Diego: Singular; 1992.

26. Johnson K. (1997). Acoustic and auditory phonetics. Londres: Blackwell, 1997.

27. Forrest K, Weismer G, Milenkovic P, Dougall RN. Statistical analysis of word-initial voiceless obstruints: preliminary data. J Acoustic Soc Am. 1988; 84:115-23.

28. Jongman A, Wayland R, Wong S. Acoustic characteristics of English fricatives. J Acoustic Soc Am. 2000; 108(3):1252-63.

29. Edwards J, Beckman ME. Methodological questions in studying consonant acquisition. Clin Linguist Phon. 2008; 22:937-56.

30. Lee S, Iverson GK. Development of stop consonants in Korean. Korean Linguistics. 2008;14:21-39.

31. Pouplier M, Goldstein L. Asymmetries in the perception of speech production errors. J Phonetics. 2005; 33:47-75.

32. Goldstein L, Pouplier M, Chen L, Saltzman E, Byrd D. Dynamic action units slip in speech production errors. Cognition. 2007; 103:386-412.
http://dx.doi.org/10.1590/S1516-18462011005000010

RECEBIDO EM: 22/07/2010

ACEITO EM: 11/09/2010

Endereço para correspondência:

Larissa Cristina Berti

Rua Antônio Lorencil Serafim, 116

Marília - SP

CEP: $17514-600$

E-mail: larissa.berti@uol.com.br 